\title{
Dynamical preparation of stripe states in spin-orbit-coupled gases
}

\author{
J. Cabedo, ${ }^{1}$ J. Claramunt, ${ }^{2}$ and A. Celi ${ }^{3}$ \\ ${ }^{1}$ Departament de Física, Universitat Autònoma de Barcelona, E-08193 Bellaterra, Spain \\ ${ }^{2}$ Department of Mathematics and Statistics, Lancaster University, Lancaster LA1 4 YW, United Kingdom \\ ${ }^{3}$ Departament de Física, Universitat Autònoma de Barcelona, 08193 Bellaterra, Spain.
}

In spinor Bose-Einstein condensates, spin-changing collisions are a remarkable proxy to coherently realize macroscopic many-body quantum states. These processes have been, e.g., exploited to generate entanglement, to study dynamical quantum phase transitions, and proposed for realizing nematic phases in atomic condensates. In the same systems dressed by Raman beams, the coupling between spin and momentum induces a spin dependence in the scattering processes taking place in the gas. Here we show that, at weak couplings, such modulation of the collisions leads to an effective Hamiltonian which is equivalent to the one of an artificial spinor gas with spin-changing collisions that are tunable with the Raman intensity. By exploiting this dressed-basis description, we propose a robust protocol to coherently drive the spin-orbit-coupled condensate into the ferromagnetic stripe phase via crossing a quantum phase transition of the effective low-energy model in an excited state.

Introduction. - Artificial spin-orbit coupling (SOC) in ultracold-atom gases offers an excellent platform for studying quantum many-body physics [1-3]. The interplay between light dressing induced by Raman coupling [4] and atom-atom interactions can lead, for instance, to high-order synthetic partial waves [5], to chiral interactions and density-dependent gauge fields [6] or to the formation of stripe phases [7]. The latter have gained significant attention over the past decade [8-13], in great part due to its supersolid like properties [14-16], that is, its simultaneous spontaneous breaking of translational invariance and of U(1) (global) phase symmetry, resulting in a crystalline structure that maintains off-diagonal long-range order.

Accessing the stripe regime of ultracold gases with SOC remains experimentally challenging, since its stability relies on the asymmetry between intra- and inter spin interactions, typically small in common spinor BoseEinstein condensates (BECs). The predicted spatial density modulations have only been unambiguously observed in [17], using orbital states in a superlattice as pseudo spin states, and very recently also in metastable states of a ${ }^{87} \mathrm{Rb}$ spinor gas [18] (for its realization in dipolar gases, see [19-21]). While sharing many properties with conventional supersolids, the nature of the stripe phases in gases with SOC is still debated [22], with current proposals focusing on probing its excitation spectrum. So far, most protocols to enhance the accessibility of the phase and the contrast of the stripes pursue an effective decrease of the intraspin interactions [23, 24]. Alternatively, here we propose an approach to access the stripe regime of a spin-1 gas with largely symmetric spin interactions, based on the coherent spin-mixing dynamics induced by Raman dressing.

Several authors have suggested a connection between spinor gases with spin-changing collisions and SOC BECs $[12,25-31]$. In this work, we show analytically that the Raman-dressed spin-1 SOC gas at low energy is equivalent, for weak Raman coupling and interactions, and zero total magnetization, to an artificial spin-1 gas with tun-
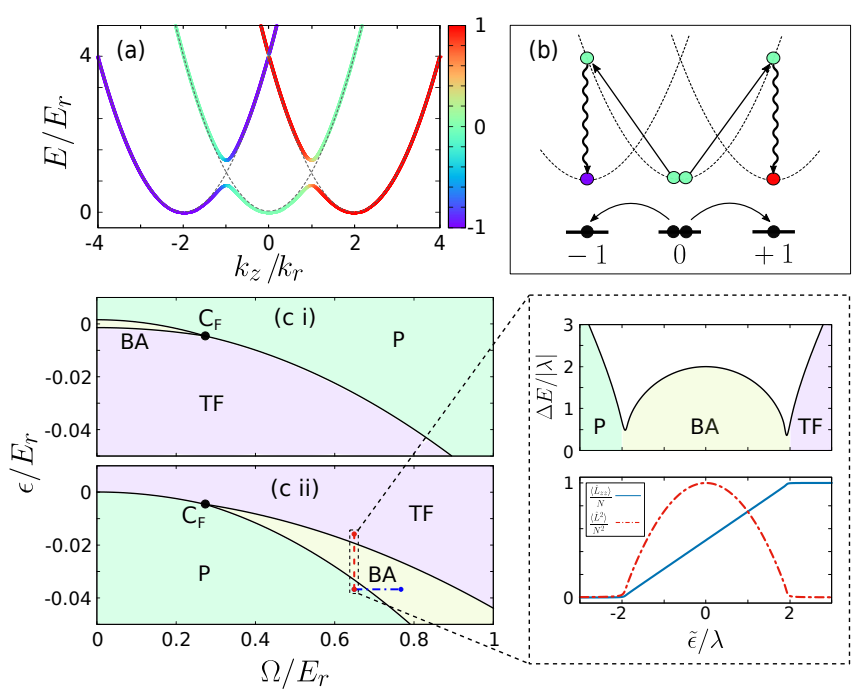

FIG. 1. (Color online) Pseudospin dynamics in SOC BECs. (a) Dispersion bands of the dressed Hamiltonian $\hat{\mathcal{H}}_{\mathrm{k}}$ with $\Omega=0.65 E_{r}, \delta=0$, and $\epsilon=\Omega^{2} / 16 E_{r}$. The color texture indicates the expected value of the spin of the dressed states. Dashed lines show the undressed dispersion bands. (b) Schematic representation of resonant collisions mediated by Raman transitions (represented by wavy lines) which act as effective spin-changing collisions. For weak Raman coupling and interactions, the dressed-state dynamics can be captured by the pseudospin Hamiltonian (4). (c i) Phase diagram of (4), as a function of the Raman Rabi frequency $\Omega$ and effective quadratic Zeeman shift $\epsilon$, for ${ }^{87} \mathrm{Rb}$ at $n=7.5 \times 10^{13} \mathrm{~cm}^{-3}$. The polar (P), twin-Fock (TF), and broken-axisymmetry (BA) phases meet at the tricritical point $C_{F}$ (black dot). (c ii) Corresponding phase diagram for the highest-excited eigenstate. The upper panel in the inset shows the energy gap between the two most excited eigenstates along the red dashed segment for $N=1000$. The lower panel shows the expected value of the collective pseudospin $\hat{L}^{2}$ (red dash-dotted line) and tensor magnetization $\hat{L}_{z z}$ (blue solid line) 
able spin-changing collisions. Under these conditions, the system is well described by a one-axis-twisting Hamiltonian [32, 33]. Such a Hamiltonian explains several quantum many-body phenomena in spinor condensates $[34,35]$, including the generation of macroscopic entanglement [36-49], with potential metrological applications [50], and the observation of nonequilibrium phenomena such as the formation of spin domains and topological defects [51-61]. Recently, dynamical [62] and excitedstate [63] quantum phase transitions have been theoretically $[64,65]$ and experimentally $[66,67]$ studied in spin- 1 BECs with spin-changing collisions. Here we exploit this map to provide a many-body protocol to access the ferromagnetic stripe phase of the SOC gas via crossing a quantum phase transition of the low-energy Hamiltonian in an excited state. This preparation enhances the accessibility of the phase, which has as the ground-state phase a very narrow region of stability [68].

System. - We consider a spin-1 Raman-dressed Bose gas held in an isotropic harmonic potential $V_{\mathrm{t}}=\frac{1}{2} m \omega_{\mathrm{t}}^{2} \boldsymbol{r}^{2}$ with the atoms interacting via two-body $s$-wave collisions. In a frame corotating and comoving with the laser beams, the system is described by the Hamiltonian $\hat{H}=\int d \boldsymbol{r}\left[\hat{\boldsymbol{\psi}}^{\dagger}\left(\hat{\mathcal{H}}_{\mathrm{k}}+V_{\mathrm{t}}\right) \hat{\boldsymbol{\psi}}+\frac{g_{0}}{2}\left(\hat{\boldsymbol{\psi}}^{\dagger} \hat{\boldsymbol{\psi}}\right)^{2}+\frac{g_{2}}{2} \sum_{j}\left(\hat{\boldsymbol{\psi}}^{\dagger} \hat{F}_{j} \hat{\boldsymbol{\psi}}\right)^{2}\right]$, with $\hat{\boldsymbol{\psi}}=\left(\hat{\psi}_{-1}, \hat{\psi}_{0}, \hat{\psi}_{1}\right)^{T}$ the spinor field operator and $\left\{\hbar \hat{F}_{x}, \hbar \hat{F}_{y}, \hbar \hat{F}_{z}\right\}$ the spin-1 matrices. Here $g_{0}=$ $4 \pi \hbar^{2}\left(a_{0}+2 a_{2}\right) / 3 m$ and $g_{2}=4 \pi \hbar^{2}\left(a_{2}-a_{0}\right) / 3 m$, with $a_{0}$ and $a_{2}$ the scattering lengths in the $F=0$ and $F=2$ channels, respectively. The dressed kinetic Hamiltonian reads $\hat{\mathcal{H}}_{\mathrm{k}}=\frac{\hbar^{2}}{2 m}\left(\boldsymbol{k}-2 k_{r} \hat{F}_{z} \boldsymbol{e}_{z}\right)^{2}+\frac{\Omega}{\sqrt{2}} \hat{F}_{x}+\delta \hat{F}_{z}+\epsilon \hat{F}_{z}^{2}$, where $\Omega$ is the Raman coupling strength, $\delta$ is the Raman detuning, and $\epsilon$ is the effective quadrupole tensor field strength. The latter term can be controlled independently of $\delta$ by employing two different Raman couplings between the two Zeeman pairs $\{|1,1\rangle,|1,0\rangle\}$ and $\{|1,0\rangle,|1,-1\rangle\}$, and simultaneously adjusting the Raman frequency differences [69]. We label the Raman single-photon recoil energy and momentum as $E_{r}=\frac{\hbar^{2} k_{r}^{2}}{2 m}$ and $\hbar k_{r}$, respectively. In the weakly coupled regime, the lowest dispersion band of $\hat{\mathcal{H}}_{\mathrm{k}}$ presents a triple-well shape along the direction of the momentum transfer, which we arbitrarily set along the $\hat{z}$ axis. Spin texture is present in the band, with the spin mixture being the largest at the vicinity of the avoided crossings (see Fig. 1(a)). While much smaller, the spin overlap between states located at the vicinity of adjacent minima is nonzero, and increases linearly with $\Omega$. This overlap allows collision processes that exchange large momentum at low energies. These Raman-mediated processes act as spin-changing collisions, as illustrated in Fig. 1(b).

Low-energy effective theory. - We now consider the regime where $\delta, \epsilon, \hbar \omega_{\mathrm{t}}$, and the interaction energy per particle are all much smaller than the recoil energy $E_{r}$. Such a low-energy landscape is well captured by an effective theory in which all the dynamics involves only the lowest band modes around each band minimum $\boldsymbol{k}_{j} \sim 2 j k_{r} \boldsymbol{e}_{z}$, with $j \in\{-1,0,1\}$. Under these considerations, we re-express the spinor field $\hat{\psi}$ in terms of the lowest-band dressed fields at the vicinity of each $\boldsymbol{k}_{j}$, which we label as $\hat{\varphi}_{j}$, and set a cut-off $\Lambda \ll \hbar k_{r}$ to the momentum spread $\boldsymbol{p}$ around them. With this notation, we can identify the operators acting in the separated regions as a pseudospinor field $\hat{\varphi}=\left(\hat{\varphi}_{-1}, \hat{\varphi}_{0}, \hat{\varphi}_{1}\right)^{T}$, with $\left[\hat{\varphi}_{i}(\boldsymbol{p}), \hat{\varphi}_{j}^{\dagger}\left(\boldsymbol{p}^{\prime}\right)\right]=\delta\left(\boldsymbol{p}-\boldsymbol{p}^{\prime}\right) \delta_{i j}$. By using perturbation theory up to second order in $\Omega$, the low-energy Hamiltonian can be written as $\hat{H} \simeq \hat{H}_{\mathrm{S}}+\hat{H}_{\mathrm{A}}$ (see the Supplemental Material for more details [70]). Here $\hat{H}_{\mathrm{S}}$ and $\hat{H}_{\mathrm{A}}$ include the pseudospin-symmetric and nonsymmetric contributions, respectively, given by

$$
\hat{H}_{\mathrm{S}}=\int d \boldsymbol{r}\left[\sum_{i} \hat{\varphi}_{i}^{\dagger}\left(\frac{\boldsymbol{p}^{2}}{2 m}+V_{\mathrm{t}}\right) \hat{\varphi}_{i}+\frac{g_{0}}{2} \sum_{i j} \hat{\varphi}_{i}^{\dagger} \hat{\varphi}_{j}^{\dagger} \hat{\varphi}_{j} \hat{\varphi}_{i}\right]
$$

and

$$
\begin{aligned}
& \hat{H}_{\mathrm{A}}=\int d \boldsymbol{r}\left[\frac{g_{2}}{2} \sum_{j}\left(\hat{\boldsymbol{\varphi}}^{\dagger} \hat{F}_{j} \hat{\boldsymbol{\varphi}}\right)^{2}+\tilde{g}_{2}\left(\hat{\varphi}_{1}^{\dagger} \hat{\varphi}_{1}+\hat{\varphi}_{-1}^{\dagger} \hat{\varphi}_{-1}\right) \hat{\varphi}_{0}^{\dagger} \hat{\varphi}_{0}\right. \\
& \left.+\tilde{g}_{2}\left(\hat{\varphi}_{1}^{\dagger} \hat{\varphi}_{-1}^{\dagger} \hat{\varphi}_{0} \hat{\varphi}_{0}+\text { H.c }\right)+\hat{\boldsymbol{\varphi}}^{\dagger}\left(\delta \hat{F}_{z}+\tilde{\epsilon} \hat{F}_{z}^{2}\right) \hat{\boldsymbol{\varphi}}\right],
\end{aligned}
$$

with $\tilde{g}_{2}=g_{0} \frac{\Omega^{2}}{16 E_{r}^{2}}$. The coefficient $\tilde{\epsilon}$ includes the correction to $\epsilon$, with $\tilde{\epsilon}=\epsilon+\frac{\Omega^{2}}{16 E_{r}}$. In (2) we have excluded the terms proportional to $g_{2} \Omega^{2}$, since typically $\left|g_{2}\right| \ll g_{0}$. Notice that, even in the case of $\mathrm{SU}(3)$-symmetric interactions (i.e. $g_{2}=0$ ), $\hat{H}_{\mathrm{A}}$ includes SOC-induced spinchanging collision processes with a spin-mixing rate $\tilde{g}_{2}$.

Three-mode model. - We now restrict ourselves to the case in which $\hat{H}_{\mathrm{A}}$ can be treated as a perturbation over the symmetric part $\hat{H}_{\mathrm{S}}$. We assume that the dynamics is then well described by a three-mode model. It includes three eigenmodes of $\hat{H}_{\mathrm{S}}$, labeled as $\left|\phi_{-1}\right\rangle,\left|\phi_{0}\right\rangle$, and $\left|\phi_{1}\right\rangle$, which have a quasi-momentum distribution centered in the vicinity of $\boldsymbol{k}_{-1}, \boldsymbol{k}_{0}$, and $\boldsymbol{k}_{1}$, respectively. By introducing the associated bosonic operators $\hat{b}_{-1}, \hat{b}_{0}$, and $\hat{b}_{1}$, we truncate the field operators to $\hat{\varphi}_{i}^{\dagger}(\boldsymbol{r}) \sim \phi_{i}^{*}(\boldsymbol{r}) \hat{b}_{i}^{\dagger}$. We call the three modes $\left|\phi_{j}\right\rangle$ pseudospin states. Finally, dropping the terms that only depend on the total number of particles $N$, we obtain the one-axis-twisting Hamiltonian

$$
\hat{H}_{\text {eff }}=\frac{\lambda}{2 N} \hat{L}^{2}-\frac{\lambda-g_{2} n}{2 N} \hat{L}_{z}^{2}+\delta \hat{L}_{z}+\tilde{\epsilon} \hat{L}_{z z},
$$

where we introduce the collective pseudospin operators $\hat{L}_{x, y, z}=\sum_{\mu \nu} \hat{b}_{\mu}^{\dagger}\left(\hat{F}_{x, y, z}\right)_{\mu \nu} \hat{b}_{\nu}$ and $\hat{L}_{z z}=\sum_{\mu \nu} \hat{b}_{\mu}^{\dagger}\left(\hat{F}_{z}^{2}\right)_{\mu \nu} \hat{b}_{\nu}$. Here $\lambda=\left(\tilde{g}_{2}+g_{2}\right) n$, where $n$ is the mean density of the gas ${ }^{1}$.

\footnotetext{
${ }^{1}$ Since the spinor modes $\left|\phi_{j}\right\rangle$ are determined through the symmetric Hamiltonian (1), we have that $\left|\phi_{i}(\boldsymbol{r})\right|=\left|\phi_{j}(\boldsymbol{r})\right|$ for all $i, j=-1,0,1$. Thus, within the subspace spanned by these three modes, the mean density of the gas is simply given by $n=N \int d \boldsymbol{r}\left|\phi_{0}(\boldsymbol{r})\right|^{4}$.
} 
Since $\left[\hat{H}_{\text {eff }}, \hat{L}_{z}\right]=0$, the total magnetization is preserved by $\hat{H}_{\text {eff }}$. Within the zero magnetization subspace (where $\hat{L}_{z}=0$ ), the effective Hamiltonian (3) reduces to

$$
\hat{H}_{0}=\lambda \frac{\hat{L}^{2}}{2 N}+\tilde{\epsilon} \hat{L}_{z z}
$$

The Hamiltonian (4) describes the nonlinear coherent spin dynamics in a spin-1 BEC, in which the densitydependent spin-symmetric interaction dominates [33]. In the SOC-based realization of (4) we propose here, we can control the spin-mixing parameter $\lambda$ independently of the density of the gas by adjusting $\Omega$. That is, SOC BECs provide an alternative platform for designing entanglement protocols and studying dynamical phase transitions.

Dynamical preparation of stripe states. - The phase diagram of the Hamiltonian (4) in the $\Omega-\epsilon$ plane is shown in Fig. 1(c i), where we use the expressions for $\lambda(\Omega)$ and $\tilde{\epsilon}(\Omega, \epsilon)$. We consider ${ }^{87} \mathrm{Rb}$, with $g_{2} / g_{0}=-0.0047[35]$ and density $n=7.5 \times 10^{13} \mathrm{~cm}^{-3}$. We now use this effective description to design a protocol to prepare dynamically the stripe phase of the dressed gas, which we later test numerically. For $\Omega>\Omega_{c}=4 E_{r} \sqrt{\left|g_{2}\right| / g_{0}}$, the diagram is equivalent to that of an antiferromagnetic spinor gas without SOC, $\lambda>0$. The ground state is then either in a polar $(\mathrm{P})$ phase, where all the atoms occupy the $\left|\phi_{0}\right\rangle$ state, or in a twin-Fock (TF) phase, in which the ground state approximates the spin- $\frac{1}{2}$ balanced Dicke state $\frac{1}{(N / 2) !}\left(\hat{b}_{-1}^{\dagger}\right)^{N / 2}\left(\hat{b}_{1}^{\dagger}\right)^{N / 2}|0\rangle$. The phase transition between the two phases is found along $\tilde{\epsilon}(\Omega)=0$. At $\Omega=\Omega_{c}$, the effective and the intrinsic spin-mixing dynamics mutually compensate, with $\tilde{g}_{2}=-g_{2}$, yielding $\lambda=0$. For $\Omega<\Omega_{c}$, the effective spin dynamics is ferromagnetic, $\lambda<0$. Then dressed spin interactions tend to maximize the total spin, resulting in a ground state with a nonvanishing transverse magnetization. This spontaneous breaking of the $\mathrm{SO}(2)$ symmetry of the system [52] gives rise to the so-called broken-axisymmetry (BA) phase [71] in between the $\mathrm{P}$ and TF phases. The two transitions take place at $\tilde{\epsilon}= \pm 2 \lambda$ in the thermodynamic limit. The three phases meet at the tricritical point $C_{F}$, at $\Omega=4 E_{r} \sqrt{\left|g_{2}\right| / g_{0}}$ and $\epsilon=g_{2} / g_{0}$.

Remarkably, the BA phase of the effective model corresponds to the super-solid like ferromagnetic stripe (FS) phase of the spin-1 SOC gas diagram, described in detail in [68]. The FS phase is characterized by the presence of spatial density modulations that are proportional to $\Omega$. When $\left|g_{2}\right|$ is small, as in ${ }^{87} \mathrm{Rb}$, such phase is only favored in a very narrow region in parameter space, which makes its experimental realization challenging. Alternatively, the ferromagnetic landscape can be probed in the most excited manifold of $\hat{H}_{0}$ in the antiferromagnetic regime, given that $\hat{H}_{0}(\lambda, \tilde{\epsilon})=-\hat{H}_{0}(-\lambda,-\tilde{\epsilon})$. In Fig. 1 (c ii) we show the phase diagram for the most excited state of $\hat{H}_{0}$. It displays the same phases as the ground state,
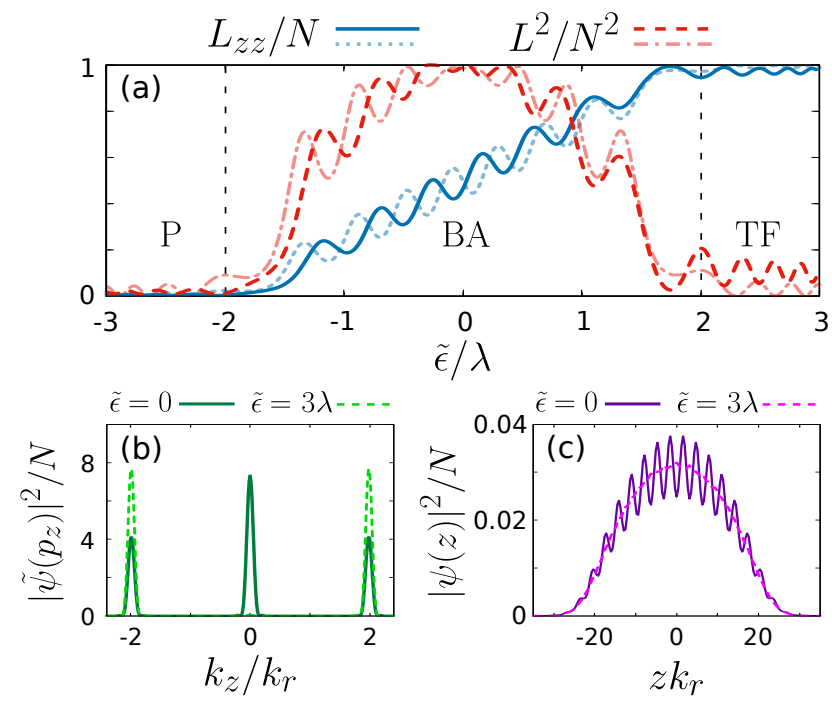

FIG. 2. (Color online) Crossing quantum phase transitions in an excited state. (a) Plot of $L_{z z}$ (blue solid line) and $L^{2}$ (red dashed line) as a function of $\tilde{\epsilon}$ for a state initially prepared at $b_{ \pm 1}=\sqrt{50}$ and $b_{0}=\sqrt{N-100}$, with $N(0)=10^{4}$ and $\hbar \omega_{\mathrm{t}}=2 \pi \times 140 \mathrm{~Hz}$. The state is evolved under the GPE while driving $\tilde{\epsilon}$ from $-3 \lambda$ to $3 \lambda$, keeping $\Omega=0.65 E_{r}$, following the red dashed path in Fig. 1(c ii). The total drive time is set to $\tau_{d}=8 h / \lambda$. The corresponding results obtained with simulations of the three-mode model (4) are shown in light colors. (b) Quasi-momentum density $\left|\tilde{\boldsymbol{\psi}}\left(p_{z}\right)\right|^{2}$ of the driven state at $\tilde{\epsilon}=0$ (dark green solid line) and $\tilde{\epsilon}=3 \lambda$ (light green dashed line). (c) Corresponding density profiles at $\tilde{\epsilon}=0$ (purple solid line) and $\tilde{\epsilon}=3 \lambda$ (pink dashed line).

but with the phase boundaries redefined. In the excitedstate diagram, the predicted BA phase occurs for a much broader range of parameters. Notably, at the P-BA and BA-TF transitions, the energy gap between the two most excited states scales weakly with the total number of particles as proportional to $\lambda N^{-1 / 3}$. This facilitates the quasi-adiabatic driving through both phase transitions in workable time scales even when the number of particles is large. This feature was exploited in [45] and [46] to generate macroscopic $\mathrm{TF}$ and BA states, respectively, in small ${ }^{87} \mathrm{Rb}$ spinor condensates.

Following the dressed-spinor description, we propose to prepare the FS phase in the most excited phase diagram of the effective model by driving an initially polarized state across the P-BA quantum phase transition therein. The loading can be easily achieved from an undressed condensate in the $m_{f}=0$ spin state by adiabatically turning up $\Omega$, while setting $\tilde{\epsilon}<-2 \lambda$. The excited phase diagram can then be probed by varying $\epsilon$ and $\Omega$. Since here the stripe phase occurs at larger $\Omega$, it exhibits a larger contrast of the density modulations, when compared to its ground-state counterpart.

To derive our protocol, we assume the validity of the three-mode truncation that leads to the Hamiltonian (4). To assess the extent of such truncation, which is 
equivalent to the single-spatial-mode approximation in spinor condensates [72], we simulate the protocol with the Gross-Pitaevskii equation (GPE) for the full dressed gas, $i \hbar \dot{\psi}_{j}=\delta \mathcal{E} / \delta \psi_{j}^{*}$, with $\mathcal{E}=\boldsymbol{\psi}^{*}\left(\hat{\mathcal{H}}_{\mathrm{k}}+V_{\mathrm{t}}\right) \boldsymbol{\psi}+\frac{g_{0}}{2}|\boldsymbol{\psi}|^{4}+$ $\frac{g_{2}}{2} \sum_{j}\left(\boldsymbol{\psi}^{*} \hat{F}_{j} \boldsymbol{\psi}\right)^{2}$, using the XMDS2 library [73] (see the Supplemental Material [70] for more details). We label the three self-consistent modes around $\boldsymbol{k}_{j}$ as $\boldsymbol{\phi}_{j}$, which are calculated via imaginary-time evolution of the GPE, and define $b_{j}=\int d \boldsymbol{r} \phi_{j}^{*}(\boldsymbol{r}) \cdot \boldsymbol{\psi}(\boldsymbol{r})$. As a reference, we consider conditions similar to those described in [46], with small ${ }^{87} \mathrm{Rb}$ condensates in the $F=1$ hyperfine manifold at $n \sim 7.5 \times 10^{13} \mathrm{~cm}^{-3}$, and take $E_{r} / \hbar=2 \pi \times 3680 \mathrm{~Hz}$, $k_{r}=7.95 \times 10^{6} \mathrm{~m}^{-1}$ and $g_{0} k_{r}^{3}=1.066 E_{r}$. Note that in the proposed protocol, the state is initially prepared in the Fock state $\frac{1}{\sqrt{N !}}\left(\hat{b}_{0}^{\dagger}\right)^{N}|0\rangle$. In these conditions, the dynamics is dominated by quantum fluctuations [74, 75] and the mean field description is expected to be inaccurate. Instead, we set the initial state to a coherent state with $0<b_{ \pm 1} \ll N$.

In Fig. 2 we show the results for a drive along the red dashed path drawn in the excited state diagram from Fig. 1(c ii). The drive is obtained with $\delta=0$, $\omega_{\mathrm{t}}=2 \pi \times 140 \mathrm{~Hz}$ and $N=10^{4}$. We set $\Omega=$ $0.65 E_{r}$, and the initial state to $b_{ \pm 1}=\sqrt{50}$ and $b_{0}=$ $\sqrt{N-100}$. In Fig. 2(a) we plot the collective pseudospin $L^{2}=\sum_{j}\left[\sum_{\mu \nu} b_{\mu}^{*}\left(\hat{F}_{j}\right)_{\mu \nu} b_{\nu}\right]^{2}$ and the tensor magnetization $L_{z z}=\sum_{\mu \nu} b_{\mu}^{*}\left(\hat{F}_{z}^{2}\right)_{\mu \nu} b_{\nu}$ as a function of $\tilde{\epsilon} / \lambda$. The state is time evolved following the linear $\operatorname{ramp} \tilde{\epsilon}(t)=$ $3 \lambda\left(2 t / \tau_{d}-1\right)$, with $\tau_{d}=8 h / \lambda$, which crosses both transitions at $\tilde{\epsilon} \sim \pm 2 \lambda$. In the BA phase, the tensor magnetization $\hat{L}_{z z}$ increases homogeneously with $\tilde{\epsilon} /|\lambda|$ and the total spin $\hat{L}$ peaks at $\tilde{\epsilon}=0$, in agreement with the effective model (see Fig.1(c)). For comparison, the results obtained from the direct simulation of the three-mode Hamiltonian (4) are shown in light colors. In Fig. 2(b) we plot the momentum-space density at the middle and at the end of the drive, in which the state approaches a BA state and a TF state, respectively. The corresponding density profiles are shown in Fig. 2(c). As expected, the excited BA phase exhibits large density modulations along the direction of the Raman beams.

Experimental considerations. - Finally, we assess the robustness of the preparation by incorporating atom loss and heating mechanisms into the simulations of the GPE. We naively model the noise in $\delta$ and $\epsilon$ with sinusoidal signals of frequency $50 \mathrm{~Hz}$ and amplitudes $300 \mathrm{~Hz}$ and $2.5 \mathrm{~Hz}$, respectively. We consider $\Omega$ to be stable during the drive, but to have a calibration uncertainty of $125 \mathrm{~Hz}$ in each realization. These amplitudes are compatible with a magnetic bias field instability of approximately $0.5 \mathrm{mG}$ and a relative uncertainty of $\pm 5 \%$ in $\Omega$, within the stabilities reached in experiments with ${ }^{87} \mathrm{Rb}$ $[7,69,76]$. At the same time we consider a $10 \%$ uncertainty in the number of atoms initially in the condensate and the population to decay as $N(t)=N(0) \exp (-\gamma t)$,


FIG. 3. (Color online) Robust preparation of FS states. (a) Plot of $L_{z z}$ (blue solid line), $L^{2}$ (red dashed line), and $f_{3 \mathrm{M}}$ (green dash-dotted line) as a function of time for a state initially prepared at $b_{ \pm 1}=\sqrt{10}$ and $b_{0}=\sqrt{N-20}$, with $N(0)=10^{4}$ and $\hbar \omega_{\mathrm{t}}=2 \pi \times 140 \mathrm{~Hz}$. The state is evolved under the GPE while driving $\tilde{\epsilon}$ from $-3 \lambda$ to 0 by linearly increasing $\Omega$ from $0.65 E_{r}$ to $0.767 E_{r}$, following the blue dashdotted path in Fig. 1(c ii). The parameters of the GPE are subject to random fluctuations that simulate experimental noise, as described in the text, and the values depicted are averaged over 20 realizations. The shadowed regions indicate the associated standard deviations. (b) Longitudinal density $|\boldsymbol{\psi}|^{2}$ (blue solid line), spin density $\mathcal{F}_{x}$ (red dashed line), and nematic density $\mathcal{N}_{x x}$ (green dash-dotted line) at $t=150 \mathrm{~ms}$ from a single realization of the drive.

with $\gamma=3.33 \mathrm{~s}^{-1}$, which is compatible with the lifetime of spin-1 Raman-dressed BECs for $\Omega<E_{r}$ [69, 77].

In these conditions, we simulate a drive following the blue dash-dotted path drawn in the excited-state diagram from Fig. 1(c ii). Along the path, $\epsilon$ is kept fixed while $\Omega$ is linearly ramped up. In this way, $\lambda$ is increased as $\tilde{\epsilon}$ approaches 0 . Such tunability of the SOC-mediated spin mixing allows one to reduce the preparation time while retaining a high robustness. At the same time, at larger $\Omega$, the contrast of the stripes is further enhanced. In Fig. 3(a), we plot $L_{z z}, L^{2}$, and the fraction of atoms that remain within the three-mode subspace, $f_{3 \mathrm{M}}=\frac{1}{N^{2}} \sum_{j}\left|b_{j}\right|^{2}$, averaged over 20 of drives. The PBA transition is well captured, with $f_{3 \mathrm{M}} \sim 0.99$ by the end of the drives. Finally, in Fig. 3(b) we plot the longitudinal density $|\boldsymbol{\psi}|^{2}$, the spin density $\mathcal{F}_{x}=\boldsymbol{\psi}^{*} \hat{F}_{x} \boldsymbol{\psi}$, and the nematic density $\mathcal{N}_{x x}=\boldsymbol{\psi}^{*}\left(\frac{2}{3}-\hat{F}_{x}^{2}\right) \boldsymbol{\psi}$ at $\tilde{\epsilon}=0$ for a single realization of the drive. As predicted by the effective model, the prepared state exhibits the characteristic properties of FS states, with large spatial modulations along the direction of the Raman beams. The FS phase can be distinguished from antiferromagnetic stripe phases from the periodicity of the modulations, with the particle density and the spin densities having periodicity $2 \pi /\left|\boldsymbol{k}_{\mathbf{1}}\right|$ and the nematic densities containing harmonic components both with period $2 \pi /\left|\boldsymbol{k}_{\mathbf{1}}\right|$ and $\pi /\left|\boldsymbol{k}_{\mathbf{1}}\right|$. As a final remark, we note that the preparation could be optimized further by employing reinforcement learning techniques, as recently demonstrated in [78].

Conclusions. - In summary, we have shown that, for weak Raman coupling and interactions, a Raman-dressed spin-1 BEC is equivalent to an artificial spinor BEC with 
tunable nonsymmetric spin interactions. A ferromagnetic gas like ${ }^{87} \mathrm{Rb}$ can be turned to antiferromagnetic by light dressing, and the stability of the FS phase is understood in these terms. We have used such insight to propose the preparation of FS phases by driving an initially polarized state through a quantum phase transition in an excited state of the Raman-dressed gas. In the excitedstate phase diagram, the FS phase is broader and both the energy gap and the density modulation contrast are larger. These features enable a robust preparation of the state and ease the detection of its supersolid properties, e.g., by probing its spectrum of excitations [11, 24].

Our dressed-base description of Raman-coupled spinor gases suggests different directions for probing nonequilibrium phenomena, as in [57, 61], with light-dressed spinor gases of alkali-metal and non-alkali-metal [79] atoms. Remarkably, the FS phase corresponds to the BA entangled phase of the artificial spinor gas: Its preparation may thus lead to the generation of macroscopic entanglement in momentum space (cf. [80]). Likewise, the map introduces SOC gases as an alternative platform to study dynamical and excited-state quantum phase transitions. The FS phase of the spin-1 gas can be understood as an excited-state quantum phase through its connection with undressed collisional spin dynamics [65]. This precise connection will be explored elsewhere [81].

We thank J. Mompart and V. Ahufinger for useful discussions and L. Tarruell for insightful discussions on experimental aspects of the Raman coupled BEC. A.C. thanks G. Juzeliunas for discussions on Raman coupled spinor BEC during his stay at Institute of Theoretical Physics and Astronomy of University of Vilnius, supported by the COST action 16221, The Quantum Technologies with Ultracold atoms. J. Cabedo and A.C. acknowledge support from the Ministerio de Economía y Competividad MINECO (Contract No. FIS201786530-P), from the European Union Regional Development Fund within the ERDF Operational Program of Catalunya (project QUASICAT/QuantumCat), and from Generalitat de Catalunya (Contract No. SGR20171646). A.C. acknowledges support from the UAB Talent Research program. J. Claramunt acknowledges partial support from the research funding Brazilian agency CAPES and the European Research Council under the European Union's Horizon 2020 research and innovation program (Grant Agreement No. 805495).

[1] J. Dalibard, F. Gerbier, G. Juzeliūnas, and P. Öhberg, "Colloquium : Artificial gauge potentials for neutral atoms," Rev. Mod. Phys. 83, 1523 (2011).

[2] N. Goldman, G. Juzeliūnas, P. Öhberg, and I. B. Spielman, "Light-induced gauge fields for ultracold atoms," Rep. Prog. Phys. 77, 126401 (2014).

[3] M. Lewenstein, A. Sanpera, and V. Ahufinger, Ultracold
Atoms in Optical Lattices: Simulating quantum manybody systems (Oxford University Press, 2012).

[4] Y.-J. Lin, R. L. Compton, K. Jiménez-García, J. V. Porto, and I. B. Spielman, "Synthetic magnetic fields for ultracold neutral atoms," Nature 462, 628 (2009).

[5] R. A. Williams, L. J. LeBlanc, K. Jimenez-Garcia, M. C. Beeler, A. R. Perry, W. D. Phillips, and I. B. Spielman, "Synthetic partial waves in ultracold atomic collisions," Science 335, 314 (2012).

[6] L. Tarruell, "Engineering chiral solitons and density-dependent gauge fields in raman-coupled bose-einstein condensates," (2020), available at https://www.youtube.com/watch?v=T1V2iIA_ewM.

[7] Y.-J. Lin, K. Jiménez-García, and I. B. Spielman, "Spin-orbit-coupled bose-einstein condensates," Nature 471, 83 (2011).

[8] C. Wang, C. Gao, C.-M. Jian, and H. Zhai, "Spin-orbit coupled spinor bose-einstein condensates," Phys. Rev. Lett. 105, 160403 (2010).

[9] T.-L. Ho and S. Zhang, "Bose-einstein condensates with spin-orbit interaction," Phys. Rev. Lett. 107, 150403 (2011).

[10] G. I. Martone, Y. Li, L. P. Pitaevskii, and S. Stringari, "Anisotropic dynamics of a spin-orbit-coupled boseeinstein condensate," Phys. Rev. A 86, 063621 (2012).

[11] Y. Li, G. I. Martone, L. P. Pitaevskii, and S. Stringari, "Superstripes and the excitation spectrum of a spin-orbitcoupled bose-einstein condensate," Phys. Rev. Lett. 110, 235302 (2013).

[12] Z. Lan and P. Öhberg, "Raman-dressed spin-1 spin-orbitcoupled quantum gas,” Phys. Rev. A 89, 023630 (2014).

[13] J. Hou, X.-W. Luo, K. Sun, T. Bersano, V. Gokhroo, S. Mossman, P. Engels, and C. Zhang, "Momentumspace josephson effects," Phys. Rev. Lett. 120, 120401 (2018).

[14] G. V. Chester, "Speculations on bose-einstein condensation and quantum crystals," Phys. Rev. A 2, 256 (1970).

[15] A. J. Leggett, "Can a solid be "superfluid"?" Phys. Rev. Lett. 25, 1543 (1970).

[16] M. Boninsegni and N. V. Prokof'ev, "Colloquium: Supersolids: What and where are they?" Rev. Mod. Phys. 84, 759 (2012).

[17] J.-R. Li, J. Lee, W. Huang, S. Burchesky, B. Shteynas, F. Ç. Top, A. O. Jamison, and W. Ketterle, "A stripe phase with supersolid properties in spin-orbit-coupled bose-einstein condensates," Nature 543, 91 (2017).

[18] A. Putra, F. Salces-Cárcoba, Y. Yue, S. Sugawa, and I. B. Spielman, "Spatial coherence of spin-orbit-coupled bose gases," Phys. Rev. Lett. 124, 053605 (2020).

[19] L. Tanzi, E. Lucioni, F. Famà, J. Catani, A. Fioretti, C. Gabbanini, R. N. Bisset, L. Santos, and G. Modugno, "Observation of a dipolar quantum gas with metastable supersolid properties," Phys. Rev. Lett. 122, 130405 (2019).

[20] F. Böttcher, J.-N. Schmidt, M. Wenzel, J. Hertkorn, M. Guo, T. Langen, and T. Pfau, "Transient supersolid properties in an array of dipolar quantum droplets," Phys. Rev. X 9, 011051 (2019).

[21] L. Chomaz, D. Petter, P. Ilzhöfer, G. Natale, A. Trautmann, C. Politi, G. Durastante, R. M. W. van Bijnen, A. Patscheider, M. Sohmen, M. J. Mark, and F. Ferlaino, "Long-lived and transient supersolid behaviors in dipolar quantum gases," Phys. Rev. X 9, 021012 (2019).

[22] J. Hofmann and W. Zwerger, "Hydrodynamics of a super- 
fluid smectic," Journal of Statistical Mechanics: Theory and Experiment 2021, 033104 (2021).

[23] J. Li, W. Huang, B. Shteynas, S. Burchesky, F. i. m. c. b. u. i. e. i. f. Top, E. Su, J. Lee, A. O. Jamison, and W. Ketterle, "Spin-orbit coupling and spin textures in optical superlattices," Phys. Rev. Lett. 117, 185301 (2016).

[24] K. T. Geier, G. I. Martone, P. Hauke, and S. Stringari, "Exciting the goldstone modes of a supersolid spin-orbitcoupled bose gas," Phys. Rev. Lett. 127, 115301 (2021).

[25] J. Higbie and D. M. Stamper-Kurn, "Generating macroscopic-quantum-superposition states in momentum and internal-state space from bose-einstein condensates with repulsive interactions," Phys. Rev. A 69, 053605 (2004).

[26] J. Lian, L. Yu, J.-Q. Liang, G. Chen, and S. Jia, "Orbit-induced spin squeezing in a spin-orbit coupled bose-einstein condensate," Sci. Rep. 3, 3166 (2013).

[27] Y. Huang and Z.-D. Hu, "Spin and field squeezing in a spin-orbit coupled bose-einstein condensate," Sci. Rep. 5, 8006 (2015).

[28] X. Y. Huang, F. X. Sun, W. Zhang, Q. Y. He, and C. P. Sun, "Spin-orbit-coupling-induced spin squeezing in three-component bose gases," Phys. Rev. A 95, 013605 (2017).

[29] J. Cabedo, J. Claramunt, A. Celi, Y. Zhang, V. Ahufinger, and J. Mompart, "Coherent spin mixing via spinorbit coupling in bose gases," Phys. Rev. A 100, 063633 (2019).

[30] L. Chen, Y. Zhang, and H. Pu, "Spin squeezing in a spin-orbit-coupled bose-einstein condensate," Phys. Rev. A 102, 023317 (2020).

[31] D. Lao, C. Raman, and C. A. R. S. de Melo, "Nematicorbit coupling and nematic density waves in spin-1 condensates," Phys. Rev. Lett. 124, 173203 (2020).

[32] M. Kitagawa and M. Ueda, "Squeezed spin states," Phys. Rev. A 47, 5138 (1993).

[33] C. K. Law, H. Pu, and N. P. Bigelow, "Quantum spins mixing in spinor bose-einstein condensates," Phys. Rev. Lett. 81, 5257 (1998).

[34] T.-L. Ho, "Spinor bose condensates in optical traps," Phys. Rev. Lett. 81, 742 (1998).

[35] D. M. Stamper-Kurn and M. Ueda, "Spinor bose gases: Symmetries, magnetism, and quantum dynamics," Rev. Mod. Phys. 85, 1191 (2013).

[36] L.-M. Duan, A. Sørensen, J. I. Cirac, and P. Zoller, "Squeezing and entanglement of atomic beams," Phys. Rev. Lett. 85, 3991 (2000).

[37] E. M. Bookjans, C. D. Hamley, and M. S. Chapman, "Strong quantum spin correlations observed in atomic spin mixing," Phys. Rev. Lett. 107, 210406 (2011).

[38] B. Lücke, M. Scherer, J. Kruse, L. Pezzé, F. Deuretzbacher, P. Hyllus, O. Topic, J. Peise, W. Ertmer, J. Arlt, L. Santos, A. Smerzi, and C. Klempt, "Twin matter waves for interferometry beyond the classical limit," Science 334, 773 (2011).

[39] C. Gross, H. Strobel, E. Nicklas, T. Zibold, N. BarGill, G. Kurizki, and M. K. Oberthaler, "Atomic homodyne detection of continuous-variable entangled twinatom states," Nature 480, 219 (2011).

[40] C. D. Hamley, C. S. Gerving, T. M. Hoang, E. M. Bookjans, and M. S. Chapman, "Spin-nematic squeezed vacuum in a quantum gas," Nat. Phys. 8, 305 (2012).

[41] Z. Zhang and L.-M. Duan, "Generation of massive entan- glement through an adiabatic quantum phase transition in a spinor condensate," Phys. Rev. Lett. 111, 180401 (2013).

[42] M. Gabbrielli, L. Pezzè, and A. Smerzi, "Spin-mixing interferometry with bose-einstein condensates," Phys. Rev. Lett. 115, 163002 (2015).

[43] I. Peise, J.and Kruse, K. Lange, B. Lücke, L. Pezzè, J. Arlt, W. Ertmer, K. Hammerer, L. Santos, A. Smerzi, and C. Klempt, "Satisfying the einstein-podolsky-rosen criterion with massive particles," Nat. Commun. 6, 8984 (2015).

[44] T. M. Hoang, H. M. Bharath, M. J. Boguslawski, M. Anquez, B. A. Robbins, and M. S. Chapman, "Adiabatic quenches and characterization of amplitude excitations in a continuous quantum phase transition," Proc. Natl. Acad. Sci. U. S. A. 113, 9475-9479 (2016).

[45] X.-Y. Luo, Y.-Q. Zou, L.-N. Wu, Q. Liu, M.-F. Han, M. K. Tey, and L. You, "Deterministic entanglement generation from driving through quantum phase transitions," Science 355, 620 (2017).

[46] Y.-Q. Zou, L.-N. Wu, Q. Liu, X.-Y. Luo, S.-F. Guo, J.-H. Cao, M. K. Tey, and L. You, "Beating the classical precision limit with spin-1 dicke states of more than 10,000 atoms," PNAS 115, 6381 (2018).

[47] P. Kunkel, M. Prüfer, H. Strobel, D. Linnemann, A. Frölian, T. Gasenzer, M. Gärttner, and M. K. Oberthaler, "Spatially distributed multipartite entanglement enables epr steering of atomic clouds," Science $\mathbf{3 6 0}$, 413 (2018).

[48] L. Pezzè, M. Gessner, P. Feldmann, C. Klempt, L. Santos, and A. Smerzi, "Heralded generation of macroscopic superposition states in a spinor bose-einstein condensate," Phys. Rev. Lett. 123, 260403 (2019).

[49] A. Qu, B. Evrard, J. Dalibard, and F. Gerbier, "Probing spin correlations in a bose-einstein condensate near the single-atom level," Phys. Rev. Lett. 125, 033401 (2020).

[50] L. Pezzè, A. Smerzi, M. K. Oberthaler, R. Schmied, and P. Treutlein, "Quantum metrology with nonclassical states of atomic ensembles," Rev. Mod. Phys. 90, 035005 (2018).

[51] J. Stenger, S. Inouye, D. M. Stamper-Kurn, H.-J. Miesner, A. P. Chikkatur, and W. Ketterle, "Spin domains in ground-state bose-einstein condensates," Nature 396, 345 (1998).

[52] L. E. Sadler, J. M. Higbie, S. R. Leslie, M. Vengalattore, and D. M. Stamper-Kurn, "Spontaneous symmetry breaking in a quenched ferromagnetic spinor bose-einstein condensate," Nature 443, 312 (2006).

[53] E. M. Bookjans, A. Vinit, and C. Raman, "Quantum phase transition in an antiferromagnetic spinor bose-einstein condensate," Phys. Rev. Lett. 107, 195306 (2011).

[54] A. Vinit, E. M. Bookjans, C. A. R. S. de Melo, and C. Raman, "Antiferromagnetic spatial ordering in a quenched one-dimensional spinor gas," Phys. Rev. Lett. 110, 165301 (2013).

[55] T. M. Hoang, M. Anquez, B. A. Robbins, X. Y. Yang, B. J. Land, C. D. Hamley, and M. S. Chapman, "Parametric excitation and squeezing in a many-body spinor condensate," Nat. Commun. 7, 11233 (2016).

[56] M. Anquez, B. A. Robbins, H. M. Bharath, M. Boguslawski, T. M. Hoang, and M. S. Chapman, "Quantum kibble-zurek mechanism in a spin-1 bose-einstein condensate," Phys. Rev. Lett. 116, 155301 (2016). 
[57] M. Prüfer, P. Kunkel, H. Strobel, S. Lannig, D. Linnemann, C.-M. Schmied, J. Berges, T. Gasenzer, and M. K. Oberthaler, "Observation of universal dynamics in a spinor bose gas far from equilibrium," Nature 563, 217 (2018).

[58] Z. Chen, T. Tang, J. Austin, Z. Shaw, L. Zhao, and Y. Liu, "Quantum quench and nonequilibrium dynamics in lattice-confined spinor condensates," Phys. Rev. Lett. 123, 113002 (2019).

[59] S. Kang, S. W. Seo, H. Takeuchi, and Y. Shin, "Observation of wall-vortex composite defects in a spinor bose-einstein condensate," Phys. Rev. Lett. 122, 095301 (2019).

[60] K. Jiménez-García, A. Invernizzi, B. Evrard, C. Frapolli, J. Dalibard, and F. Gerbier, "Spontaneous formation and relaxation of spin domains in antiferromagnetic spin-1 condensates," Nat. Commun. 10, 1422 (2019).

[61] M. Prüfer, T. V. Zache, P. Kunkel, S. Lannig, A. Bonnin, H. Strobel, J. Berges, and M. K. Oberthaler, "Experimental extraction of the quantum effective action for a non-equilibrium many-body system," Nat. Phys. 16, 1012 (2020).

[62] M. Heyl, "Dynamical quantum phase transitions: a review," Rep. Prog. Phys. 81, 054001 (2018).

[63] P. Cejnar, P. Stránský, M. Macek, and M. Kloc, "Excited-state quantum phase transitions," J. Phys. A: Math. Theor. 54, 133001 (2021).

[64] C. B. Dă̆, S.-T. Wang, and L.-M. Duan, "Classification of quench-dynamical behaviors in spinor condensates," Phys. Rev. A 97, 023603 (2018).

[65] P. Feldmann, C. Klempt, A. Smerzi, L. Santos, and M. Gessner, "Interferometric order parameter for excitedstate quantum phase transitions in bose-einstein condensates," Phys. Rev. Lett. 126, 230602 (2021).

[66] H.-X. Yang, T. Tian, Y.-B. Yang, L.-Y. Qiu, H.-Y. Liang, A.-J. Chu, C. B. Dağ, Y. Xu, Y. Liu, and L.-M. Duan, "Observation of dynamical quantum phase transitions in a spinor condensate," Phys. Rev. A 100, 013622 (2019).

[67] T. Tian, H.-X. Yang, L.-Y. Qiu, H.-Y. Liang, Y.-B. Yang, $\mathrm{Y} . \mathrm{Xu}$, and L.-M. Duan, "Observation of dynamical quantum phase transitions with correspondence in an excited state phase diagram," Phys. Rev. Lett. 124, 043001 (2020).

[68] G. I. Martone, F. V. Pepe, P. Facchi, S. Pascazio, and S. Stringari, "Tricriticalities and quantum phases in spinorbit-coupled spin-1 bose gases," Phys. Rev. Lett. 117, 125301 (2016).

[69] D. L. Campbell, R. M. Price, A. Putra, A. Valdés-Curiel, D. Trypogeorgos, and I. B. Spielman, "Magnetic phases of spin-1 spin-orbit-coupled bose gases," Nat. Commun. 7, 10897 (2016).

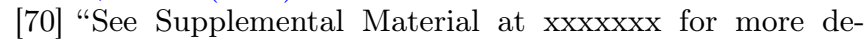
tails.".

[71] K. Murata, H. Saito, and M. Ueda, "Brokenaxisymmetry phase of a spin-1 ferromagnetic boseeinstein condensate," Phys. Rev. A 75, 013607 (2007).

[72] S. Yi, O. E. Müstecaplığlu, C. P. Sun, and L. You, "Single-mode approximation in a spinor-1 atomic condensate," Phys. Rev. A 66, 011601 (2002).

[73] G. R. Dennis, J. J. Hope, and M. T. Johnsson, "Xmds2: Fast, scalable simulation of coupled stochastic partial differential equations," Comput. Phys. Commun. 184, 201-208 (2013).

[74] C. Klempt, O. Topic, G. Gebreyesus, M. Scherer, T. Hen- ninger, P. Hyllus, W. Ertmer, L. Santos, and J. J. Arlt, "Parametric amplification of vacuum fluctuations in a spinor condensate," Phys. Rev. Lett. 104, 195303 (2010).

[75] B. Evrard, A. Qu, J. Dalibard, and F. Gerbier, "Coherent seeding of the dynamics of a spinor bose-einstein condensate: From quantum to classical behavior," Phys. Rev. A 103, L031302 (2021).

[76] X.-T. Xu, Z.-Y. Wang, R.-H. Jiao, C.-R. Yi, W. Sun, and S. Chen, "Ultra-low noise magnetic field for quantum gases," Rev. Sci. Instrum. 90, 054708 (2019).

[77] R. P. Anderson, D. Trypogeorgos, A. Valdés-Curiel, Q.-Y. Liang, J. Tao, M. Zhao, T. Andrijauskas, G. Juzeliūnas, and I. B. Spielman, "Realization of a deeply subwavelength adiabatic optical lattice," Phys. Rev. Research 2, 013149 (2020).

[78] S.-F. Guo, F. Chen, Q. Liu, M. Xue, J.-J. Chen, J.-H. Cao, T.-W. Mao, M. K. Tey, and L. You, "Faster state preparation across quantum phase transition assisted by reinforcement learning," Phys. Rev. Lett. 126, 060401 (2021).

[79] T. Chalopin, T. Satoor, A. Evrard, V. Makhalov, J. Dalibard, R. Lopes, and S. Nascimbene, "Probing chiral edge dynamics and bulk topology of a synthetic hall system," Nat. Phys. 16, 1017 (2020).

[80] F. Anders, A. Idel, P. Feldmann, D. Bondarenko, S. Loriani, K. Lange, J. Peise, M. Gersemann, B. Meyer, S. Abend, N. Gaaloul, C. Schubert, D. Schlippert, L. Santos, E. Rasel, and C. Klempt, "Momentum entanglement for atom interferometry," (2020), arXiv:2010.15796 [quant-ph].

[81] J. Cabedo and A. Celi, "Excited-state quantum phase transitions in spin-orbit coupled bose gases," (2021), arXiv:2109.01495 [cond-mat.quant-gas]. 


\section{Supplemental Material}

In this supplementary document we include the detailed derivation of the low-energy Hamiltonian introduced in the main text. We also provide additional insights on the approach taken to assess the validity of the three-state model derived, and on its robustness.

\section{EFFECTIVE LOW-ENERGY THEORY}

Here we detail the derivation of the effective low-energy theory presented in the main text for weakly-coupled Raman-dressed spin-1 BECs, with Rabi frequency $\Omega<1$ (in units of recoil energy). We restrict ourselves to a regime in which the linear and quadratic Zeeman terms, denoted by $\delta$ and $\epsilon$ respectively, are also small, and set $|\delta|$, $|\epsilon| \ll 1$. In this regime, the low-energy landscape only involves the dressed states located around the three minima of the dispersion band. Thus, we set a cut-off $\Lambda \ll 1$ (in units of $k_{r}$ ) to the momentum spread $p$ around each minimum, so that $|p|<\Lambda$. Under these conditions, we use second order perturbation theory to express the bare fields $\hat{\psi}_{i}$ in terms of the lowest-band dressed-state fields $\hat{\varphi}_{j}$ around the center band minimum

$$
\begin{gathered}
\hat{\psi}_{0}(p)=\left(1-\frac{\Omega^{2}}{64}\left(1-\frac{\epsilon}{2}+O\left(\left(\Lambda+\frac{\epsilon+\delta}{4}\right)^{2}\right)\right)\right) \hat{\varphi}_{0}(p)+O\left(\left(\frac{\Omega}{8(1-\Lambda)}\right)^{3}\right), \\
\hat{\psi}_{ \pm 1}(p)=-\frac{\Omega}{8}\left(1-\frac{\epsilon \pm \delta \mp 4 p}{4}+O\left(\left(\Lambda+\frac{\epsilon+\delta}{4}\right)^{2}\right)\right) \hat{\varphi}_{0}(p)+O\left(\left(\frac{\Omega}{8(1-\Lambda)}\right)^{3}\right)
\end{gathered}
$$

and in right/left band minima

$$
\begin{aligned}
\hat{\psi}_{ \pm 1}( \pm 2+p) & =\left(1-\frac{1}{2}\left(\frac{\Omega}{8}\right)^{2}\left(1+\frac{\epsilon \pm \delta \mp 4 p}{2}+O\left(\left(\Lambda+\frac{\epsilon+\delta}{4}\right)^{2}\right)\right)\right) \hat{\varphi}_{ \pm 1}(p)+O\left(\left(\frac{\Omega}{8(1-\Lambda)}\right)^{3}\right) \\
\hat{\psi}_{0}( \pm 2+p) & =-\frac{\Omega}{8}\left(1+\frac{\epsilon \pm \delta \mp 4 p}{4}+O\left(\left(\Lambda+\frac{\epsilon+\delta}{4}\right)^{2}\right)\right) \hat{\varphi}_{ \pm 1}(p)+O\left(\left(\frac{\Omega}{8(1-\Lambda)}\right)^{3}\right) \\
\hat{\psi}_{\mp 1}( \pm 2+p) & =\frac{\Omega^{2} / 16}{\left(\left(16+p^{2} \pm \delta \pm 8 p\right)\left(1-\frac{\epsilon \mp \delta \pm 4 p}{4}\right)\right.} \hat{\varphi}_{ \pm 1}(p)+O\left(\left(\frac{\Omega}{8(1-\Lambda)}\right)^{3}\right)
\end{aligned}
$$

respectively. We made explicit only the dependence on momentum along the direction of the recoil momentum transfer. Notice that the positions of the edge band minima are actually shifted from \pm 2 by a small amount proportional to $\Omega^{2}$. Still, up to second order in $\Omega$, these shifts do not contribute to expressions (S2), and hence are not included. Note that the last term of the above expressions can be neglected since it contributes to the interactions at fourth order in $\frac{\Omega}{8(1-\Lambda)}$. As shown below, due to momentum conservation, the nontrivial contributions to the interacting Hamiltonian involve only the first order terms in the above expressions, while the second order just renormalize the symmetric interactions.

We adopt the notation short cuts

$$
\iint \hat{\psi}_{a}^{\dagger} \hat{\psi}_{b}^{\dagger} \hat{\psi}_{a} \hat{\psi}_{b} \equiv \frac{g}{2} \int \mathrm{d} r \int \prod_{j=1}^{4} \frac{\mathrm{d}^{3} k_{j}}{(2 \pi)^{3}} e^{i \mathbf{r} \cdot\left(\mathbf{k}_{1}+\mathbf{k}_{2}-\mathbf{k}_{3}-\mathbf{k}_{4}\right)} \hat{\psi}_{a}^{\dagger}\left(\mathbf{k}_{1}\right) \hat{\psi}_{b}^{\dagger}\left(\mathbf{k}_{2}\right) \hat{\psi}_{a}\left(\mathbf{k}_{3}\right) \hat{\psi}_{b}\left(\mathbf{k}_{4}\right),
$$

and

$$
\iint \hat{\varphi}_{a}^{\dagger} \hat{\varphi}_{b}^{\dagger} \hat{\varphi}_{a} \hat{\varphi}_{b} \equiv \frac{g}{2} \int \mathrm{d} r \int_{-\Lambda}^{\Lambda} \prod_{j=1}^{4} \frac{\mathrm{d}^{3} p_{j}}{(2 \pi)^{3}} e^{i \mathbf{r} \cdot\left(\mathbf{p}_{1}+\mathbf{p}_{2}-\mathbf{p}_{3}-\mathbf{p}_{4}\right)} \hat{\varphi}_{a}^{\dagger}\left(\mathbf{p}_{1}\right) \hat{\varphi}_{b}^{\dagger}\left(\mathbf{p}_{2}\right) \hat{\varphi}_{a}\left(\mathbf{p}_{3}\right) \hat{\varphi}_{b}\left(\mathbf{p}_{4}\right) .
$$

When the interaction operators are evaluated on the low-energy states, it follows that

$$
\begin{aligned}
\iint \hat{\psi}_{ \pm}^{\dagger} \hat{\psi}_{ \pm}^{\dagger} \hat{\psi}_{ \pm} \hat{\psi}_{ \pm}= & \iint\left(1-\frac{\Omega^{2}}{32}\left(1+\frac{\epsilon \pm \delta}{2} \mp \frac{p_{1}+p_{2}+p_{3}+p_{4}}{2}+O\left(\left(\Lambda+\frac{\epsilon+\delta}{4}\right)^{2}\right)\right)\right) \hat{\varphi}_{ \pm}^{\dagger} \hat{\varphi}_{ \pm}^{\dagger} \hat{\varphi}_{ \pm} \hat{\varphi}_{ \pm} \\
& +\frac{\Omega^{2}}{16} \iint\left(1-\frac{\epsilon \pm \delta}{2} \pm\left(p_{2}+p_{4}\right)+O\left(\left(\Lambda+\frac{\epsilon+\delta}{4}\right)^{2}\right)\right) \hat{\varphi}_{ \pm}^{\dagger} \hat{\varphi}_{0}^{\dagger} \hat{\varphi}_{ \pm} \hat{\varphi}_{0}
\end{aligned}
$$




$$
\begin{aligned}
\iint \hat{\psi}_{0}^{\dagger} \hat{\psi}_{0}^{\dagger} \hat{\psi}_{0} \hat{\psi}_{0}= & \iint\left(1-\frac{\Omega^{2}}{16}\left(1-\frac{\epsilon}{2}+O\left(\left(\Lambda+\frac{\epsilon+\delta}{4}\right)^{2}\right)\right)\right) \hat{\varphi}_{0}^{\dagger} \hat{\varphi}_{0}^{\dagger} \hat{\varphi}_{0} \hat{\varphi}_{0} \\
& +\frac{\Omega^{2}}{16} \iint\left(1+\frac{\epsilon+\delta}{2}-\left(p_{1}+p_{3}\right)+O\left(\left(\Lambda+\frac{\epsilon+\delta}{4}\right)^{2}\right)\right) \hat{\varphi}_{+}^{\dagger} \hat{\varphi}_{0}^{\dagger} \hat{\varphi}_{+} \hat{\varphi}_{0} \\
& +\frac{\Omega^{2}}{16} \iint\left(1+\frac{\epsilon-\delta}{2}+\left(p_{1}+p_{3}\right)+O\left(\left(\Lambda+\frac{\epsilon+\delta}{4}\right)^{2}\right)\right) \hat{\varphi}_{-}^{\dagger} \hat{\varphi}_{0}^{\dagger} \hat{\varphi}_{-} \hat{\varphi}_{0} \\
& +\frac{\Omega^{2}}{32} \iint\left(1+\frac{\epsilon}{2}-\left(p_{1}-p_{2}\right)+O\left(\left(\Lambda+\frac{\epsilon+\delta}{4}\right)^{2}\right)\right) \hat{\varphi}_{+}^{\dagger} \hat{\varphi}_{-}^{\dagger} \hat{\varphi}_{0} \hat{\varphi}_{0} \\
& +\frac{\Omega^{2}}{32} \iint\left(1+\frac{\epsilon}{2}-\left(p_{3}-p_{4}\right)+O\left(\left(\Lambda+\frac{\epsilon+\delta}{4}\right)^{2}\right)\right) \hat{\varphi}_{0}^{\dagger} \hat{\varphi}_{0}^{\dagger} \hat{\varphi}_{+} \hat{\varphi}_{-},
\end{aligned}
$$

$$
\begin{aligned}
\iint \hat{\psi}_{ \pm}^{\dagger} \hat{\psi}_{0}^{\dagger} \hat{\psi}_{ \pm} \hat{\psi}_{0}= & \iint\left(1-\frac{\Omega^{2}}{64}\left(3-\frac{\epsilon \mp \delta}{2} \mp\left(p_{1}+p_{3}\right)+O\left(\left(\Lambda+\frac{\epsilon+\delta}{4}\right)^{2}\right)\right)\right) \hat{\varphi}_{ \pm}^{\dagger} \hat{\varphi}_{0}^{\dagger} \hat{\varphi}_{ \pm} \hat{\varphi}_{0} \\
& +\frac{\Omega^{2}}{64} \iint\left(1-\frac{\epsilon \pm \delta}{2} \pm\left(p_{1}+p_{3}\right)+O\left(\left(\Lambda+\frac{\epsilon+\delta}{4}\right)^{2}\right)\right) \hat{\varphi}_{0}^{\dagger} \hat{\varphi}_{0}^{\dagger} \hat{\varphi}_{0} \hat{\varphi}_{0} \\
& +\frac{\Omega^{2}}{64} \iint\left(1 \pm\left(p_{1}-p_{2}\right)+O\left(\left(\Lambda+\frac{\epsilon+\delta}{4}\right)^{2}\right)\right) \hat{\varphi}_{0}^{\dagger} \hat{\varphi}_{ \pm}^{\dagger} \hat{\varphi}_{ \pm} \hat{\varphi}_{0} \\
& +\frac{\Omega^{2}}{64} \iint\left(1 \pm\left(p_{3}-p_{4}\right)+O\left(\left(\Lambda+\frac{\epsilon+\delta}{4}\right)^{2}\right)\right) \hat{\varphi}_{0}^{\dagger} \hat{\varphi}_{ \pm}^{\dagger} \hat{\varphi}_{0} \hat{\varphi}_{ \pm} \\
& +\frac{\Omega^{2}}{64} \iint\left(1+\frac{\epsilon \pm \delta}{2} \mp\left(p_{2}+p_{4}\right)+O\left(\left(\Lambda+\frac{\epsilon+\delta}{4}\right)^{2}\right)\right) \hat{\varphi}_{ \pm}^{\dagger} \hat{\varphi}_{ \pm}^{\dagger} \hat{\varphi}_{ \pm} \hat{\varphi}_{ \pm} \\
& +\frac{\Omega^{2}}{64} \iint\left(1+\frac{\epsilon \mp+\delta}{2} \pm\left(p_{2}+p_{4}\right)+O\left(\left(\Lambda+\frac{\epsilon+\delta}{4}\right)^{2}\right)\right) \hat{\varphi}_{ \pm}^{\dagger} \hat{\varphi}_{\mp}^{\dagger} \hat{\varphi}_{ \pm} \hat{\varphi}_{\mp} \\
& +\frac{\Omega^{2}}{64}\left(1 \mp \delta / 2 \pm\left(p_{2}+p_{3}\right)+O\left(\left(\Lambda+\frac{\epsilon+\delta}{4}\right)^{2}\right)\right) \hat{\varphi}_{ \pm}^{\dagger} \hat{\varphi}_{\mp}^{\dagger} \hat{\varphi}_{0} \hat{\varphi}_{0} \\
& +\frac{\Omega^{2}}{64}\left(1 \mp \delta / 2 \pm\left(p_{1}+p_{4}\right)+O\left(\left(\Lambda+\frac{\epsilon+\delta}{4}\right)^{2}\right)\right) \hat{\varphi}_{0}^{\dagger} \hat{\varphi}_{0}^{\dagger} \hat{\varphi}_{ \pm} \hat{\varphi}_{\mp},
\end{aligned}
$$

$$
\begin{aligned}
\iint \hat{\psi}_{ \pm}^{\dagger} \hat{\psi}_{\mp}^{\dagger} \hat{\psi}_{ \pm} \hat{\psi}_{\mp}= & \iint\left(1-\frac{\Omega^{2}}{32}\left(1+\frac{\epsilon}{2} \mp \frac{p_{1}-p_{2}+p_{3}-p_{4}}{2}+O\left(\left(\Lambda+\frac{\epsilon+\delta}{4}\right)^{2}\right)\right)\right) \hat{\varphi}_{ \pm}^{\dagger} \hat{\varphi}_{\mp}^{\dagger} \hat{\varphi}_{ \pm} \hat{\varphi}_{\mp} \\
& +\frac{\Omega^{2}}{64} \iint\left(1-\frac{\epsilon \pm \delta}{2} \pm\left(p_{1}+p_{3}\right)+O\left(\left(\Lambda+\frac{\epsilon+\delta}{4}\right)^{2}\right)\right) \hat{\varphi}_{0}^{\dagger} \hat{\varphi}_{\mp}^{\dagger} \hat{\varphi}_{0} \hat{\varphi}_{\mp} \\
& +\frac{\Omega^{2}}{64} \iint\left(1-\frac{\epsilon \mp \delta}{2} \mp\left(p_{2}+p_{4}\right)+O\left(\left(\Lambda+\frac{\epsilon+\delta}{4}\right)^{2}\right)\right) \hat{\varphi}_{ \pm}^{\dagger} \hat{\varphi}_{0}^{\dagger} \hat{\varphi}_{ \pm} \hat{\varphi}_{0} \\
& +\frac{\Omega^{2}}{64} \iint\left(1-\frac{\epsilon}{2} \pm\left(p_{1}-p_{2}\right)+O\left(\left(\Lambda+\frac{\epsilon+\delta}{4}\right)^{2}\right)\right) \hat{\varphi}_{0}^{\dagger} \hat{\varphi}_{0}^{\dagger} \hat{\varphi}_{ \pm} \hat{\varphi}_{\mp} \\
& +\frac{\Omega^{2}}{64} \iint\left(1-\frac{\epsilon}{2} \pm\left(p_{3}-p_{4}\right)+O\left(\left(\Lambda+\frac{\epsilon+\delta}{4}\right)^{2}\right)\right) \hat{\varphi}_{ \pm}^{\dagger} \hat{\varphi}_{\mp}^{\dagger} \hat{\varphi}_{0} \hat{\varphi}_{0} .
\end{aligned}
$$


Inserting (S5)-(S8) into the symmetric contribution to the interacting Hamiltonian $\hat{V}_{s}$, we get

$$
\begin{aligned}
\hat{V}_{s} & =\iint\left(\sum_{a=-1,0,+1}\left(\hat{\psi}_{a}^{\dagger} \hat{\psi}_{a}^{\dagger} \hat{\psi}_{a} \hat{\psi}_{a}+2 \sum_{b>a} \hat{\psi}_{a}^{\dagger} \hat{\psi}_{b}^{\dagger} \hat{\psi}_{a} \hat{\psi}_{b}\right)\right) \\
& =\iint\left(\sum_{a=-1,0,+1}\left(\hat{\varphi}_{a}^{\dagger} \hat{\varphi}_{a}^{\dagger} \hat{\varphi}_{a} \hat{\varphi}_{a}+2 \sum_{b>a} \hat{\varphi}_{a}^{\dagger} \hat{\varphi}_{b}^{\dagger} \hat{\varphi}_{a} \hat{\varphi}_{b}\right)\right) \\
& +\frac{\Omega^{2}}{8} \iint\left(\left(\hat{\varphi}_{+1}^{\dagger} \hat{\varphi}_{+1}+\hat{\varphi}_{-1}^{\dagger} \hat{\varphi}_{-1}\right) \hat{\varphi}_{0}^{\dagger} \hat{\varphi}_{0}+\left(\hat{\varphi}_{+1}^{\dagger} \hat{\varphi}_{-1}^{\dagger} \hat{\varphi}_{0} \hat{\varphi}_{0}+\text { H.c. }\right)+O\left(\left(\Lambda+\frac{\epsilon+\delta}{4}\right)^{2}\right)\right) \\
& +\frac{\Omega^{2}}{16} \iint\left(\left(p_{2}-p_{1}+p_{4}-p_{3}\right)\left(\hat{\varphi}_{+1}^{\dagger} \hat{\varphi}_{0}^{\dagger} \hat{\varphi}_{+1} \hat{\varphi}_{0}-\hat{\varphi}_{-1}^{\dagger} \hat{\varphi}_{0}^{\dagger} \hat{\varphi}_{-1} \hat{\varphi}_{0}+\left(\hat{\varphi}_{+1}^{\dagger} \hat{\varphi}_{-1}^{\dagger} \hat{\varphi}_{0} \hat{\varphi}_{0}+\text { H.c. }\right)\right)+O\left(\left(\Lambda+\frac{\epsilon+\delta}{4}\right)^{2}\right)\right) .
\end{aligned}
$$

The last term in (S9) contains a correction to the spin-mixing contribution that depends linearly on the momentum. However, its value is bounded by the cutoff in the momentum spread around the wells. Since $\left|p_{i}-p_{j}\right|<2 \Lambda \ll 1$, for simplicity we neglect such correction to the interacting Hamiltonian.

Finally, considering that, for $|p|>\Lambda$, the fields $\hat{\varphi}_{j}(p)$ vanish when acting on the low energy subspace, we can formally remove the cut-off in the integration and perform the Fourier transform. By doing so, we obtain the expression introduced in the main text for the symmetric interacting Hamiltonian in the dressed basis, namely

$$
\hat{V}_{s}=\int d \boldsymbol{r}\left[\frac{g_{0}}{2} \sum_{i j} \hat{\varphi}_{i}^{\dagger} \hat{\varphi}_{j}^{\dagger} \hat{\varphi}_{j} \hat{\varphi}_{i}+\tilde{g}_{2}\left(\hat{\varphi}_{1}^{\dagger} \hat{\varphi}_{1}+\hat{\varphi}_{-1}^{\dagger} \hat{\varphi}_{-1}\right) \hat{\varphi}_{0}^{\dagger} \hat{\varphi}_{0}+\tilde{g}_{2}\left(\hat{\varphi}_{1}^{\dagger} \hat{\varphi}_{-1}^{\dagger} \hat{\varphi}_{0} \hat{\varphi}_{0}+\hat{\varphi}_{1} \hat{\varphi}_{-1} \hat{\varphi}_{0}^{\dagger} \hat{\varphi}_{0}^{\dagger}\right)\right],
$$

with $\tilde{g}_{2}=g_{0} \frac{\Omega^{2}}{16}\left(1+O\left(\left(\Lambda+\frac{\epsilon+\delta}{4}\right)^{2}\right)\right)$. Proceeding analogously with the nonsymmetric part of the interaction potential, $\hat{V}_{a}=\frac{g_{2}}{2} \int d \boldsymbol{r} \sum_{j}\left(\hat{\boldsymbol{\psi}}^{\dagger} \hat{F}_{j} \hat{\boldsymbol{\psi}}\right)^{2}$, yields corrections to Hamiltonian (2) in the main text of the order $g_{2} \Omega^{2}$, which are safely neglected since $\left|g_{2}\right| \ll g_{0}$ for ${ }^{87} \mathrm{Rb}$.

\section{MEAN-FIELD SIMULATIONS OF THE THREE-MODE MODEL}

In the protocol described in the main text, the state approaches the Fock states $\frac{1}{\sqrt{N !}}\left(\hat{b}_{0}^{\dagger}\right)^{N}|0\rangle$ and $\frac{1}{(N / 2) !}\left(\hat{b}_{-1}^{\dagger}\right)^{N / 2}\left(\hat{b}_{1}^{\dagger}\right)^{N / 2}|0\rangle$ while being in the $\mathrm{P}$ and TF phases, respectively. The mean field description of the evolution away from the BA phase is therefore expected to be inaccurate, with the dynamics being dominated by quantum
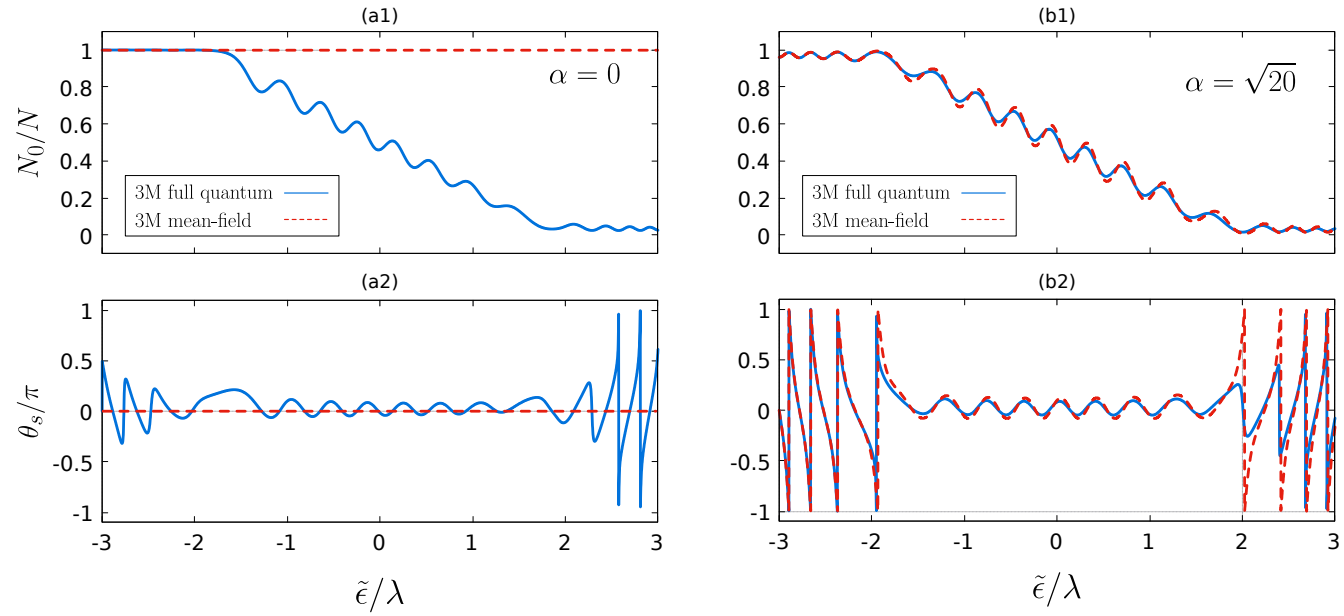

FIG. S1. (Color online) Comparison between full quantum and mean-field simulations. A state initially prepared at $|N, \alpha, 0\rangle$ is driven from $\tilde{\epsilon}=-3 \lambda$ to $\tilde{\epsilon}=3 \lambda$ with $\tau_{d}=8 h / \lambda$. The relative occupation of the state $\left|\phi_{0}\right\rangle, N_{0} / N$, along the drive is plotted in (a1) and (b1) for $\alpha=0$ and $\alpha=\sqrt{20}$, respectively. In both cases $N=1000$. The corresponding spinor phase $\theta_{s}$ is plotted in (a2) and (b2). Solid blue lines show the results from full quantum simulations of Hamiltonian (S11). Red dashed lines show the results obtained with the mean-field equations (S12). 
fluctuations. Expressing Hamiltonian (4) in the main text explicitly in terms of the mode operators $\hat{b}_{j}$ yields

$$
\hat{H}_{0}=\frac{\lambda}{N}\left[\left(\hat{b}_{-1}^{\dagger} \hat{b}_{1}^{\dagger} \hat{b}_{0} \hat{b}_{0}+\text { H.c. }\right)+\hat{N}_{0}\left(\hat{N}_{1}+\hat{N}_{-1}\right)\right]-\tilde{\epsilon} \hat{N}_{0}
$$

From eq. (S11), the corresponding three-mode mean-field equations read

$$
\begin{aligned}
i \hbar \dot{b}_{1} & =\frac{\lambda}{N}\left[b_{-1}^{*} b_{0} b_{0}+b_{0}^{*} b_{0} b_{1}\right], \\
i \hbar \dot{b}_{0} & =\frac{\lambda}{N}\left[2 b_{1} b_{-1} b_{0}^{*}+b_{1}^{*} b_{1} b_{0}+b_{-1}^{*} b_{-1} b_{0}\right]-\tilde{\epsilon} b_{0}, \\
i \hbar \dot{b}_{-1} & =\frac{\lambda}{N}\left[b_{1}^{*} b_{0} b_{0}+b_{0}^{*} b_{0} b_{-1}\right],
\end{aligned}
$$

where we have identified $\left\langle\hat{b}_{ \pm 1,0}\right\rangle=b_{ \pm 1,0}$. Initially setting $b_{ \pm 1}=0$ or $b_{0}=0$ into eqs. (S12) results in a stationary state, independently of $\tilde{\epsilon}$, in contradiction with the dynamics predicted by Hamiltonian (S11). To address this issue, we test the effective model with the GPE of the full gas by simulating an analogous drive across the P-TF-BA excited diagram in a slightly lower lying family of excited states. As shown in [65], the properties of the excited phases of Hamiltonian (4) in the main text vary smoothly across the energy spectrum. Therefore, we instead prepare the initial state in a coherent state $\left|N, \alpha, \theta_{s}\right\rangle=\frac{1}{\sqrt{N !}}\left(\alpha e^{-i \theta_{s} / 2} \hat{b}_{-1}^{\dagger}+\sqrt{1-2 \alpha^{2}} \hat{b}_{0}^{\dagger}+\alpha e^{-i \theta_{s} / 2} \hat{b}_{1}^{\dagger}\right)^{N}|0\rangle$, averaging $\alpha^{2}>0$ atoms in the pseudospin \pm 1 states. In these conditions, mean-field computations quickly converge to full quantum simulations as $\alpha$ is increased, as exemplified in Fig. S1, while the energy gap and the location of the phase boundaries do not vary significantly as long as $\alpha^{2} \ll N$.

\section{VALIDITY OF THE THREE-MODE APPROXIMATION}

The realization of stripe phases in an excited state permits to access the phase in regimes where it is experimentally more feasible. Notably, for nearly spin-symmetric BECs, the approach enhances the contrast of the spatial modulations in gas, which is proportional to $\Omega$. Since the protocol presented in the main text relies on the effective description of
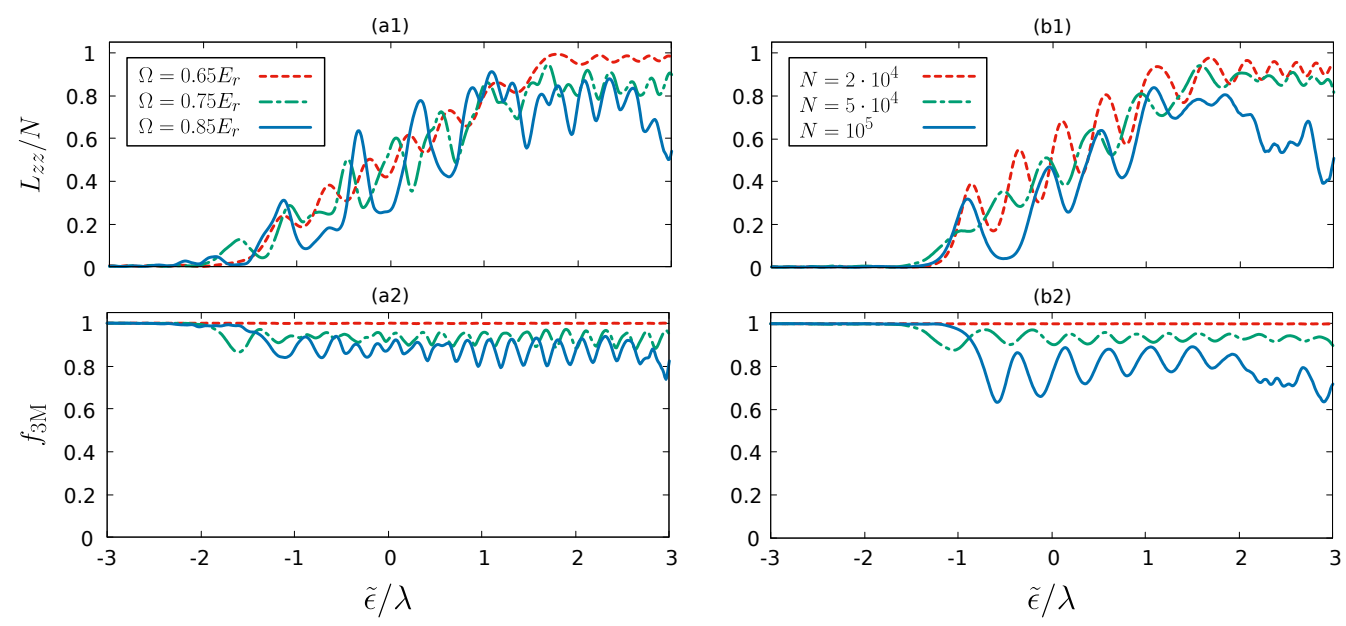

FIG. S2. (Color online) Validity of the three-mode approximation. (a1) Expected value of $\hat{L}_{z z}$ as a function of $\tilde{\epsilon}$ for a state initially prepared at $\boldsymbol{\psi}=\sqrt{\alpha}\left(\phi_{-1}+\phi_{1}\right)+\sqrt{N-2 \alpha} \phi_{0}$, with $N=10^{4}, \alpha=25$ and $\hbar \omega_{\mathrm{t}}=2 \pi \cdot 140 \mathrm{~Hz}$. The state is evolved under the GPE while driving $\tilde{\epsilon}$ from $-3 \lambda$ to $3 \lambda$, and keeping $\Omega=0.65 E_{r}$ (red dashed line), $\Omega=0.75 E_{r}$ (green dash-dotted line) and $\Omega=0.85 E_{r}$ (solid blue). The total drive time is set to $\tau_{d}=8 h / \lambda$. (a2) Relative occupation of the three self-consistent modes $\phi_{-1}, \phi_{0}$ and $\phi_{1}$, along the drive depicted in (a1). (b1) Expected value of $\hat{L}_{z z}$ as a function of $\tilde{\epsilon}$ for a state initially prepared at $\boldsymbol{\psi}=\alpha\left(\phi_{-1}+\phi_{1}\right)+\sqrt{N-2 \alpha^{2}} \phi_{0}$, with $\alpha=\sqrt{10^{-3} N}$ and $N=2 \cdot 10^{4}$ (dashed-red), $N=5 \cdot 10^{4}$ (green dash-dotted line) and $N=8 \cdot 10^{4}$ (solid blue). The state is evolved under the GPE, driving $\tilde{\epsilon}$ from $-3 \lambda$ to $3 \lambda$, while keeping $\Omega=0.5 E_{r}$ and adjusting $\omega_{\mathrm{t}}$ so that $n=10^{-14} \mathrm{~cm}^{-3}$. The total drive time is set to $\tau_{d}=8 h / \lambda$. (b2) Corresponding relative occupation of the modes $\phi_{-1}, \phi_{0}$ and $\phi_{1}$ along the drive depicted in (b1). 
Hamiltonian (4) in the main text, we discuss here its validity. Hamiltonian (4) follows from a three-mode truncation of the Hilbert space, and it predicts the energy gap that is exploited in the quasi-adiabatic protocol to drive the state through a quantum phase transition. Qualitatively, the approximation is expected to be accurate for small condensates when $|\lambda|,|\tilde{\epsilon}| \ll g_{0} n, \hbar \omega_{\mathrm{t}}$. Nonetheless, it is difficult to quantitatively estimate its accuracy. To this end, we use the GPE of the full dressed and trapped spinor gas, and quantifies the accuracy of the approximation by computing the projection of the time-evolved states on to the subspace spanned by the three self-consistent mode, $f_{3 \mathrm{M}}=\frac{1}{N^{2}} \sum_{j}\left|\int d \boldsymbol{r} \boldsymbol{\phi}_{j}^{*} \cdot \boldsymbol{\psi}\right|^{2}$, previously computed via imaginary time evolution.

In the main text, we exemplify the realization of the protocol with simulated drives along two different trajectories in the $\Omega-\epsilon$ plane of the most excited phase diagram of the effective three-mode model. In both cases, the trajectories start at $\Omega=0.65$, and we set $N(0)=10^{4}$. In general, with $\tilde{g_{2}} \propto \Omega^{2}$, the energy scale of the effective model is enhanced at larger $\Omega$, which reduces the preparation time and the relative impact of the heating mechanisms and of photon scattering loss. However, the validity of the three-mode model is progressively more challenged as $\Omega$ is increased. Similarly, at any given density, the robustness of the protocol strongly depends on the number of particles, as exemplified in Fig. S2.

Note that different physical quantities are affected differently by the value of $f_{3 \mathrm{M}}$. For instance, macroscopic entanglement preparation is expected to be very sensitive to the full quantum structure of the prepared state. Thus, even tiny reduction of $f_{3 \mathrm{M}}$ are expected to result in considerable reduction of entanglement. On the contrary, the macroscopic spin transfer is less sensitive to the the leakage of probability amplitude out of the three-mode description, as exemplified in Fig. S2. As a consequence, the optimal experimental parameters will be strongly dependent on the physical observables of interest. 